\section{Arthrose: Aktivitätsbezo- gene Belastungen lösen häufig Schub im Knie aus}

Thomas MJ et al. Triggers for acute flare in adults

with, or at risk of, knee osteoarthritis: a web-based case-crossover study in community-dwelling

adults. Osteoarthritis Cartilage 2021; 29: 956-964

Schübe mit Schmerzen können für Patienten mit Arthrose belastend sein und die Lebensqualität beeinträchtigen. Zudem führen sie häufig zu Arbeitsausfällen und einer erhöhten Inanspruchnahme des Gesundheitswesens. Ein fundiertes Verständnis der Ursachen von Krankheitsschüben ist für Patienten und Ärzte wichtig, um die Auswirkungen zu verhindern oder zu minimieren. Thomas et al. identifizierten konsistente Auslöser für Arthrose-Schübe im Knie.

Die Forscher der englischen Arbeitsgruppe kamen zu dem Resultat, dass zahlreiche aktivitätsbezogene, psychosoziale und umweltbedingte Belastungen an der Auslösung von Krankheitsschüben beteiligt sind. Insbesondere eine erhöhte körperliche Aktivität löste einen Arthrose-Schub aus. Die Forscher schlossen über 13 Wochen Erwachsene mit einem Alter von 40 Jahren mit Wohnsitz in England in die Studie ein, die an Knieschmerzen mit oder ohne dokumentierter Kniearthrose-Diagnose litten. Die eingeschlossenen Patienten erhielten ein Online-Konto auf der Studienwebsite, über die sie einen BaselineFragebogen, vier Follow-Up-Fragebögen und einen ereignisinitiierten Fragebogen bei auftretenden Knieschmerzen ausfüllten. Anhand des Baseline-Fragebogens erhoben die Forscher Informationen zu einzelnen Aspekten der Knieschmerzen, zur Inanspruchnahme von medizinischer Versorgung bei Knieschmerzen, zum allgemeinen Gesundheitszustand, zum Maß an körperlicher Aktivität und zu demografischen Merkmalen. Die Follow-Up-Fragebögen, die die Studienteilnehmer in den Wochen 1, 5, 9 und 13 nach dem Ausfüllen des Baseline-Fragebogens ausfüllten, enthielten eine Matrix, über die die Exposition gegenüber 21 mutmaßlichen aktivitätsbezogenen, psychosozialen und umweltbedingten Auslösern erfasst wurde. Patienten mit einem akuten Schub baten die Wissenschaftler, einen ereignisbezogenen Fragebogen auszufüllen, mit dem sie über mehrere Tage hinweg bis zur Auflösung des Schubs Informationen zur Schmerzintensität, Beeinträchtigungen, Medikamenteneinnahme und zur Einschätzung der Patienten hinsichtlich der Dauer des Schubs sammelten.

Zwischen Juli 2018 und Februar 2019 rekrutierten die Forscher 744 Patienten. Insgesamt 568 ereignisbezogene und 867 reguläre Fragebögen konnten die Experten für die statistische Analyse nutzen. Insbesondere Kniebeugen, Hocken oder Knien standen in positivem Zusammenhang mit dem Auftreten eines Schubes innerhalb von 24 Stunden nach der körperlichen Aktivität. Langes Sitzen ohne Pause, eine Reduktion oder ein Aussetzen einer Medikation und Husten, eine Erkältung oder leichte Infektionen reduzierten die Wahrscheinlichkeit für einen Arthrose-Schub des Knies. Treppensteigen, Autofahren, stressige Ereignisse am Arbeitsplatz oder zu Hause sowie Stress im Freundes- und Familienkreis standen in keinem statistisch signifikanten Zusammenhang mit dem Auftreten von Schüben. Während einem Schub litten die Patienten vor allem an Steifheit (64\%), Hinken (58\%), zunehmende Schwierigkeiten bei alltäglichen Aktivitäten (57\%), Schlafstörungen (48\%) und Schwellungen (33\%). In höherem Alter traten Schübe seltener auf, Frauen und Personen, die zu Studienbeginn häufig schwere Knieschmerzen hatten, waren häufiger von Schüben betroffen. $70 \%$ der Teilnehmer gaben an, dass die Schübe unerwartet auftraten. Im Mittel dauerte ein Schub 5 Tage.

\section{FAZIT}

Die Autoren identifizierten in vorliegender Studie mehrere Faktoren, die bei Erwachsenen mit Knie-Arthrose innerhalb von 24 Stunden einen akuten Schub auslösen können. Insbesondere Kniebeugen, Hocken und Knien sind mit dem Auftreten eines Schubes assoziiert. Diese Erkenntnisse können Patienten und Ärzten helfen, gemeinsam einen Schub der Knie-Arthrose besser vorherzusagen, zu verhindern und zu behandeln, so die Experten.

Dr. Maddalena Angela Di Lellis, Tübingen 